\title{
miR-145 inhibits tumor growth and metastasis by targeting metadherin in high-grade serous ovarian carcinoma
}

\author{
Ruifen Dong ${ }^{1}$, Xiaolin Liu ${ }^{1}$, Qing Zhang ${ }^{1}$, Zhijun Jiang ${ }^{1}$, Yingwei Li ${ }^{1}$, Yuyan Wei ${ }^{1}$, \\ Yinuo Li ${ }^{1}$, Qifeng Yang ${ }^{4}$, Jinsong Liu ${ }^{6}$, Jian-Jun $\mathrm{Wei}^{5}$, Changshun Shao ${ }^{3}$, \\ Zhaojian Liu' ${ }^{2}$ Beihua Kong ${ }^{1}$ \\ ${ }^{1}$ Department of Obstetrics and Gynecology, Qilu Hospital, Shandong University, Jinan, Shandong, China \\ ${ }^{2}$ Department of Cell Biology, Shandong University School of Medicine, Jinan, Shandong, China \\ ${ }^{3}$ Ministry of Education Key Laboratory of Experimental Teratology and Department of Molecular Medicine and Genetics, \\ Shandong University School of Medicine, Jinan, China \\ ${ }^{4}$ Department of Breast Surgery, Qilu Hospital, Shandong University, Jinan, Shandong, China \\ ${ }^{5}$ Department of Pathology, Northwestern University School of Medicine, Chicago, IL, USA \\ ${ }^{6}$ Department of Pathology, The University of Texas MD Anderson Cancer Center, Houston, TX, USA \\ Correspondence to:
}

Beihua Kong, e-mail: kongbeihua@sdu.edu.cn

Zhaojian Liu, e-mail: liujian9782@sdu.edu.cn

Key words: miR-145, MTDH, p53, HGSOC, metastasis

Received: September 18, 2014

Accepted: September 25, 2014

Published: October 23, 2014

\section{ABSTRACT}

High-grade serous ovarian carcinoma (HGSOC), the most common and aggressive subtype of epithelial ovarian cancer, is characterized by TP53 mutations and genetic instability. Using miRNA profiling analysis, we found that miR-145, a p53 regulated miRNA, was frequently down-regulated in HGSOC. miR-145 down-regulation was further validated in a large cohort of HGSOCs by qPCR. Overexpression of miR-145 in ovarian cancer cells significantly suppressed proliferation, migration and invasion in vitro and inhibited tumor growth and metastasis in vivo. Metadherin (MTDH) was subsequently identified as a direct target of miR-145, and was found to be significantly up-regulated in HGSOC. Furthermore, overexpression of MTDH rescued the inhibitory effects of miR-145 in ovarian cancer cells. Finally, we found that high level of MTDH expression correlated with poor prognosis of HGSOC. Therefore, lack of suppression of MTDH by miR-145 when p53 is dysfunctional leads to increased tumor growth and metastasis of HGSOC. Our study established a new link between p53, miR-145 and MTDH in the regulation of tumor growth and metastasis in HGSOC.

\section{INTRODUCTION}

Epithelial ovarian cancer is the most lethal gynecologic malignancy with several histological subtypes. High-grade serous ovarian carcinoma (HGSOC or HGSC), the most common and aggressive subtype, has the highest mortality rate, with a 5-year survival rate of only $30 \%[1]$. The advance stage at the time of diagnosis and relapse due to chemoresistance are the major reasons for poor prognosis[1]. HGSOC is characterized by TP53 mutations and genetic instability[2]. According to The Cancer Genome Atlas (TCGA), TP53 and BRCA1/2 mutations are the major genetic alterations occuring in $96 \%$ and $22 \%$ of HGSOC cases, respectively [3]. A genetically defined mouse model which closely mimic to human HGSOC was established recently by inactivating BRCA1, p53 and PTEN genes[4]. Despite those encouraging developments, the pathogenesis of HGSOC remains poorly understood and no biomarkers for prediction of response to therapy are yet available in clinical.

miRNAs are found to play important roles in tumorigenesis and metastasis in human cancers $[5,6]$. Recent reports showed that miRNAs were aberrantly expressed in HGSOC[7]. We previously showed that miR-182 was overexpressed in HGSOC and miR-182 
overexpression conferred a potent oncogenic property by targeting BRCA1 and MTSS1[8]. Using miRNA expression profiling in HGSOC, we found that a p53 regulated miRNA, miR-145, was down-regulated in most HGSOC cases. miR-145 expression level has been shown to be decreased in various human cancers such as breast cancer [9] and ovarian cancer[7]. miR-145 was reported to inhibit tumor angiogenesis and metastasis in various cancers by targeting several protein coding genes such as c-myc and N-cadherin[10-12]. However, the pathobiological significance of aberrant miR-145 expression in HGSOC has not been fully elucidated.

In the current study, we investigated the functional role of miR-145 in HGSOC, both in vitro and in vivo. We found that overexpression of miR-145 in ovarian cancer cells significantly suppressed proliferation, migration and invasion in vitro and inhibited tumor growth and metastasis in vivo. Furthermore, metadherin (MTDH), an oncogene that was highly expressed in breast cancer[13] and ovarian cancer[14], was identified as a direct target of miR-145.

\section{RESULTS}

\section{Down-regulation of miR-145 in HGSOC tissues and ovarian cancer cell lines}

The Cancer Genome Atlas (TCGA) project showed that TP53 mutations were detected in almost all HGSOC (96\%)[3]. Since p53 regulates the expression of many miRNAs[15], we speculated that p53 mutations may contribute to the tumorigenesis and metastasis of HGSOC through p53 regulated miRNAs. To identify aberrantly expressed miRNAs in HGSOC, we conducted a global miRNA expression analysis in HGSOC and normal fallopian tube fimbria (five cases each, Figure 1A, Table S1). We found that one of p53 regulated miRNAs, miR-145, was down-regulated in HGSOC. This finding revealed by microarray profiling was confirmed with qPCR (Figure 1B). Three significantly up-regulated miRNAs identified in our previous study were also examined, indicating the reliability of current profile data. To determine whether miR-145 was down-regulated in most HGSOC, we examined miR145 expression level in a larger sample. As shown in Figure 1C, miR-145 was significantly down-regulated in HGSOC $(n=48)$ compared with fimbria $(n=19)$. We further measured miR-145 expression in six ovarian cancer cell lines and one immortalized fallopian tube epithelial cell line. We found that the expression levels of miR-145 in ovarian cancer cell lines were much lower than in normal cell line (Figure 1D). These results indicate that miR-145 is down-regulated in both HGSOC tissues and cancer cell lines.

\section{Overexpression of miR-145 suppresses proliferation of ovarian cancer cells in vitro}

To investigate the possible role of miR-145 in ovarian cancer, we established three ovarian cancer cell lines with stable miR-145 overexpression (Figure 2A). We then measured the inhibitory effect of miR-145 on ovarian cancer cell proliferation. As shown in Figure 2B-D, overexpression of miR-145 significantly suppressed the proliferation of ovarian cancer cells as measured by MTT assay. The inhibitory effect of miR-145 on ovarian cancer cell proliferation was further evaluated by clonogenic assay. As shown in Figure 2E-F, the colonyforming efficiency was significantly reduced in miR-145 overexpressed cells than in control cells. These findings suggest that miR-145 suppresses proliferation of ovarian cancer cells in vitro.

\section{miR-145 suppresses migration and invasion of ovarian cancer cells in vitro}

To test whether miR-145 overexpression suppresses tumor cell migration and invasion, we first examined the morphological changes in miR-145 overexpressed cells. As shown in Figure 3A, A2780 and SKOV3 cells overexpressing miR-145 exhibited epithelial morphology. The migration and invasion were then measured by transwell assay. We found that miR-145 could significantly suppress migration (Figure 3B) and invasion (Figure 3C) in all three ovarian cancer cells lines. To further investigate whether the inhibitory effect of miR-145 on migration and invasion was mediated by mesenchyme to epithelia transition (MET), we examined the expression of several MET markers. As expected, miR-145 overexpression increased the expression level of epithelial marker (E-cadherin) and decreased the levels of mesenchymal markers (Figure 3D). Taken together, these findings suggest that miR-145 could impede invasion mediated by MET in vitro.

\section{miR-145 inhibits tumor growth and metastasis of xenografts}

We next examined whether miR-145 overexpression could suppress ovarian cancer tumor growth and metastasis in vivo, in nude mice. A2780 ovarian cancer cells with and without miR-145 overexpression were subcutaneously inoculated in nude mice $(\mathrm{n}=5$ for each group). We found that the tumor sizes derived from A2780-miR-145 overexpressing cells were smaller than those in control group A2780-NC (Figure 4A and 4B). Additionally, the tumors formed from the A2780miR-145 cells weighed significantly less when compared to A2780-NC (Figure 4C). To examine tumor metastasis, we injected, via tail vein, SKOV3 cells with and without 
A
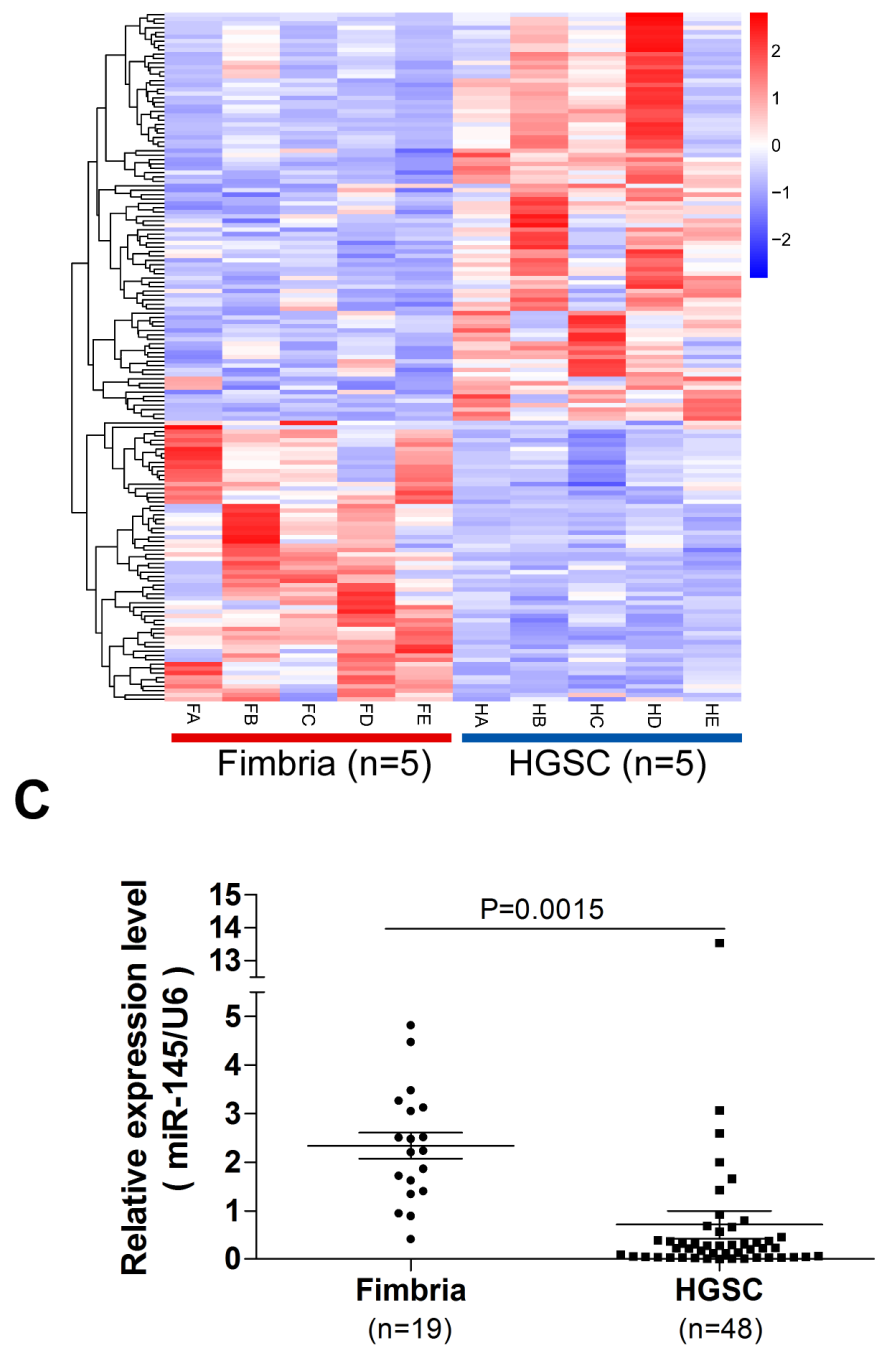

B

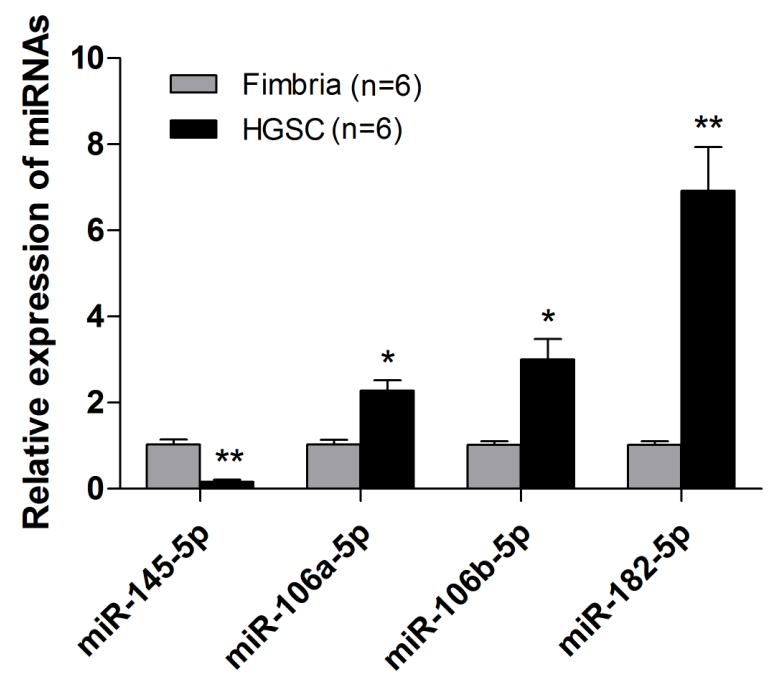

D

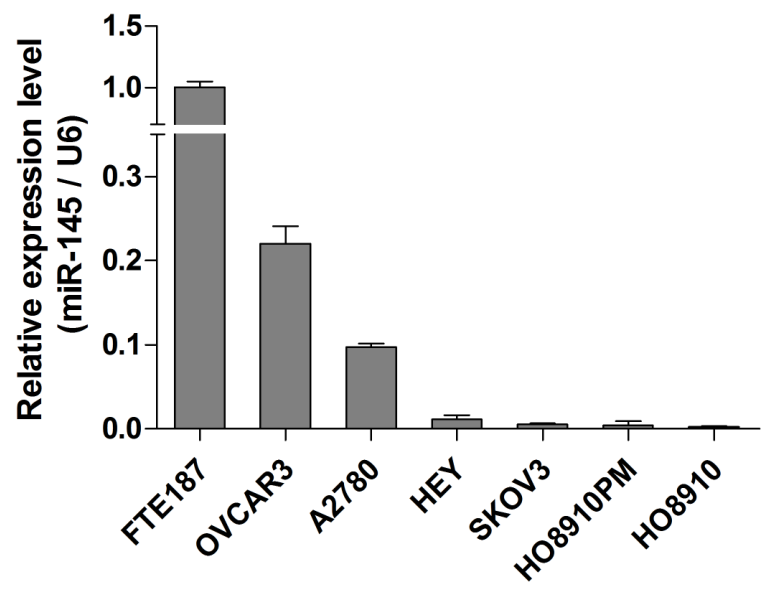

Figure 1: Down-regulation of miR-145 in ovarian cancer tissues and cell lines. (A) Heat map illustrates the dysregulated miRNAs in HGSOC compared with normal fimbria according to the five pairs of tissue microarray results. HA-HE represent the 5 high grade serous cancer samples, and FA-FE represent the 5 normal fimbria samples. (B) Validation of four microRNAs differential expression in normal fimbria and HGSOC with qPCR assay. (C) QPCR validation of miR-145 expression in fresh fimbria and HGSOC in a large cohort. (D) Relative miR-145 expression levels of six ovarian cancer cell lines and one normal fallopian tube epithelial cell line were examined with qPCR assay. $* \mathrm{P}<0.05, * * \mathrm{P}<0.01$.

miR-145 overexpression into nude mice. After 6 weeks mice were anesthetized and mouse lungs were harvested, fixed and dissected. Four out of five mice transplanted with SKOV3-NC cells had visible pulmonary metastatic nodules, while no mouse in the SKOV3-miR-145 group showed visible lung metastasis (Figure 4D). These results suggest that overexpression of miR-145 inhibits tumor growth and metastasis in vivo.

\section{MTDH is a direct target gene of miR-145}

To identify the functional target of miR-145 in HGSOC, we analyzed proteins that were up-regulated in HGSOC and the predicted targets of miR-145 from publicly available databases as PicTar, TargetScan and miRDB using Venn diagram (data not shown). MTDH was among the predicted target genes of miR-145. MTDH was reported to be overexpressed in breast cancer and to be associated with poor prognosis, due to its participation in tumorigenesis, metastasis and chemoresistance $[13,16]$. To investigate whether MTDH contributes to HGSOC, we first examined the MTDH expression in ovarian cancer cell lines and primary HGSOC tissues. We found that MTDH level was significantly higher in ovarian cancer cells than in normal epithelial cells (Figure 5A). Consistently, MTDH showed much higher level of protein 
A

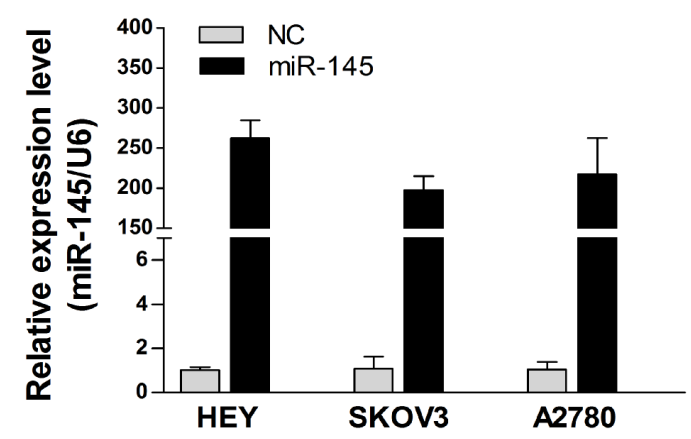

C
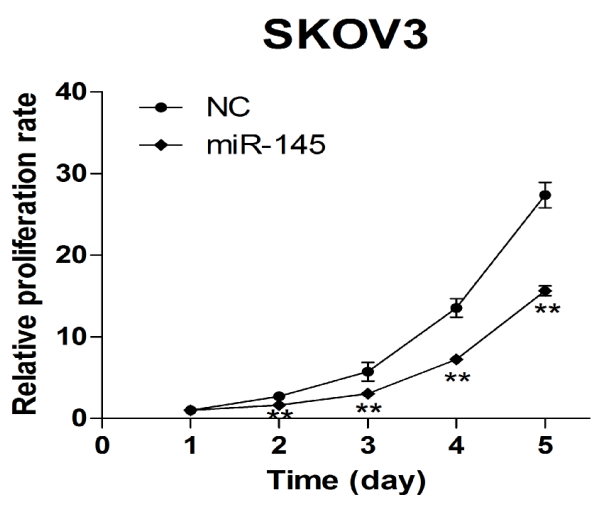

E
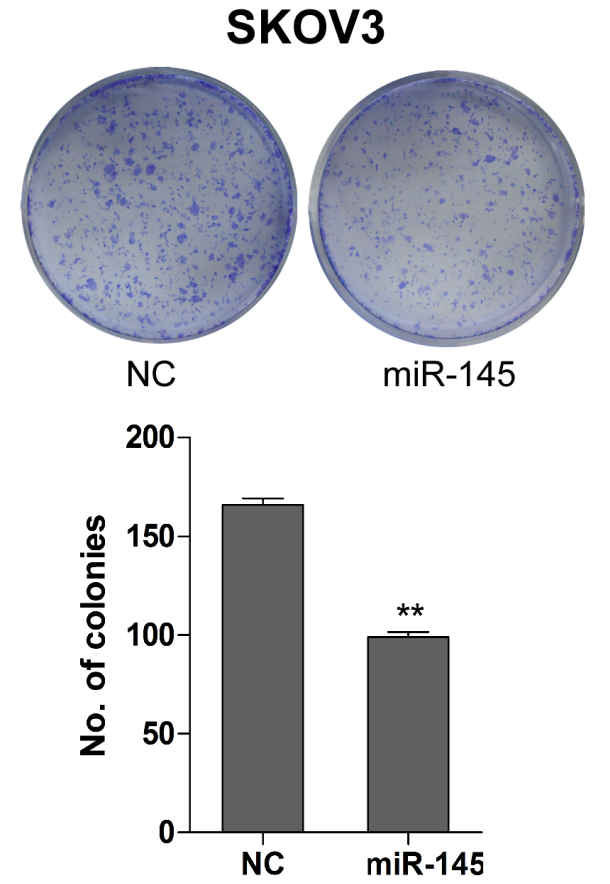

B

HEY

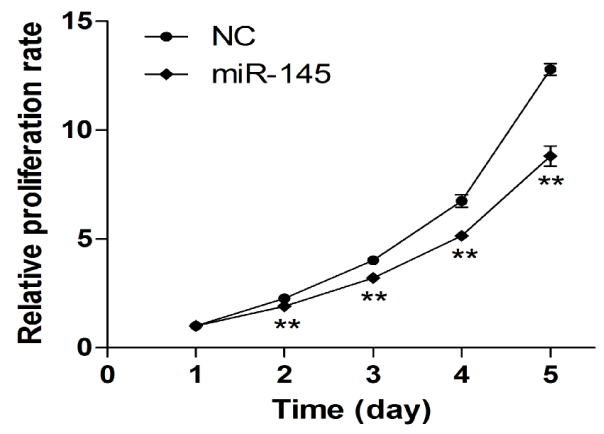

D

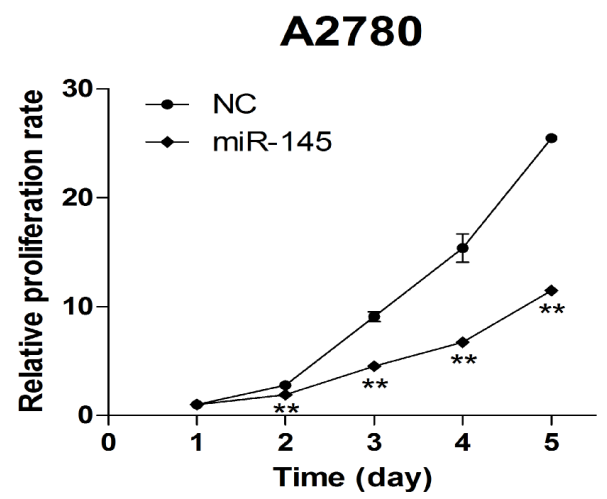

F
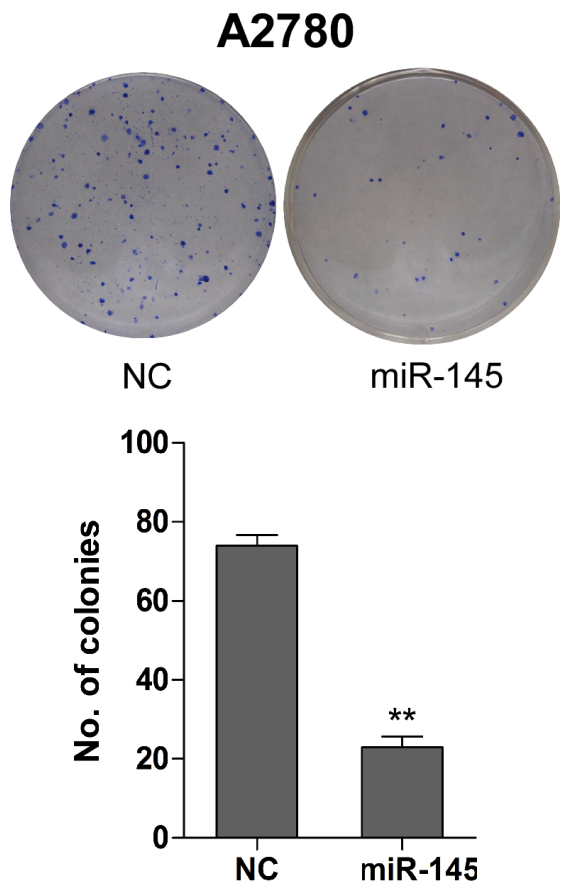

Figure 2: Stable miR-145 overexpression suppresses cell proliferation and clonogenic ability in vitro. (A) The overexpression of miR-145 in HEY, SKOV3 and A2780 cells was validated with qPCR. (B-D) Effects of ectopic expression of miR-145 on the proliferation of HEY, SKOV3 and A2780 cells were examined with MTT assay and shown as growth curve. (E-F) Effects of overexpression of miR-145 on the clonogenic ability of SKOV3 and A2780 cells. The upper panel represents dishes by colony formation assay, and the lower panel illustrates the number of colonies formed. Columns: average of three independent experiments; bars: se. ${ }^{* *} \mathrm{P}<0.01$. 


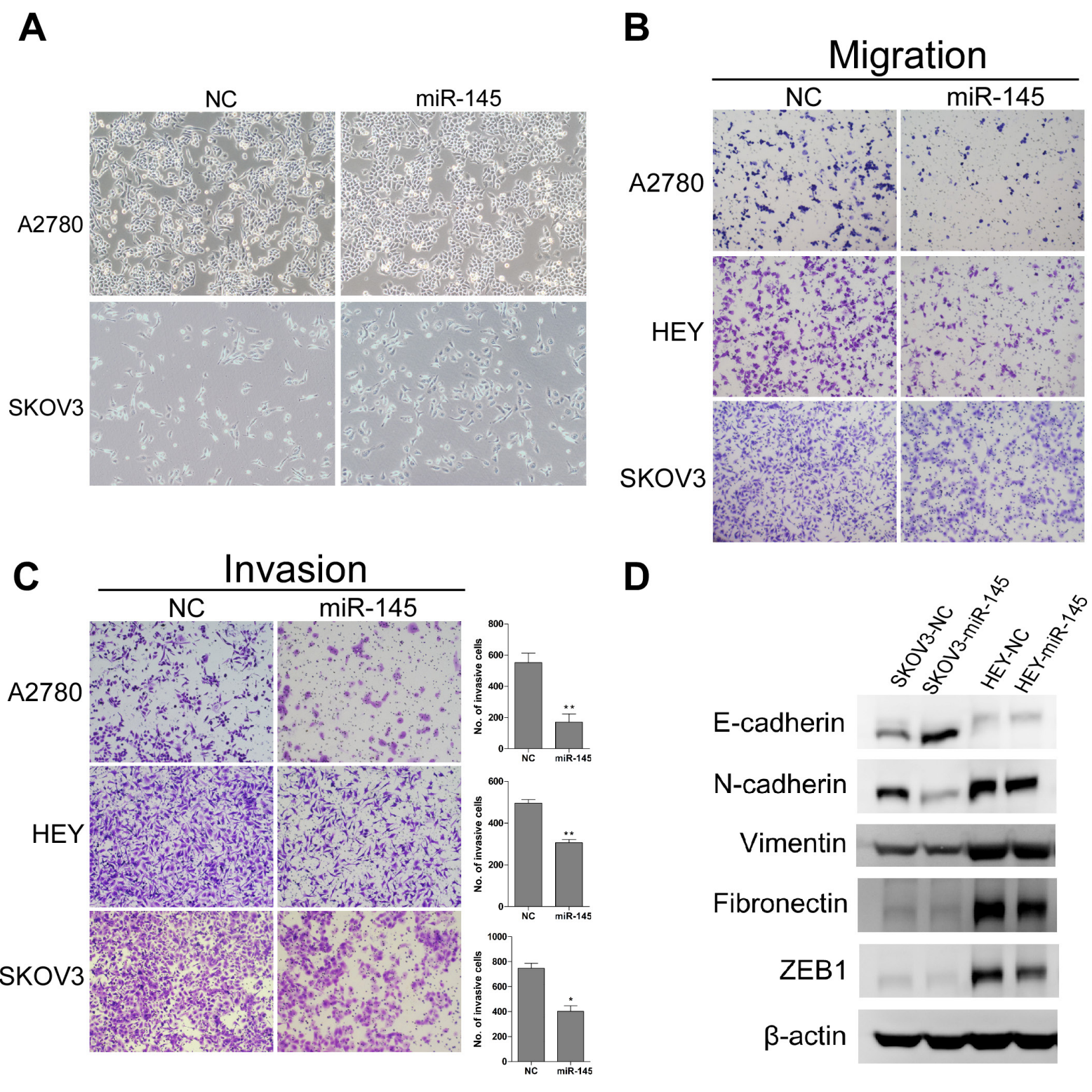

Figure 3: Ectopic expression of miR-145 suppresses ovarian cancer cell migration and invasion in vitro and induced mesenchymal to epithelial transformation(MET). (A) Morphological changes in miR-145 overexpressed cells compared to control cells. (B) Effects of ectopic expression of miR-145 on the migration of A2780, HEY and SKOV3 cells. (C) Effects of ectopic expression of miR-145 on the invasion of A2780, HEY and SKOV3 cells. (D) MET related markers show different expression in SKOV3 and HEY cells with and without miR-145 overexpression. bars: se. ${ }^{*} \mathrm{P}<0.05, * * \mathrm{P}<0.01$.

expression in primary HGSOC tissues( $\mathrm{n}=48)$ compared to normal fimbria $(n=13)$ by western bolt (Figure $5 B)$. We next tested whether MTDH could be directly targeted by miR-145. There are two possible binding sites for miR-145 in the 3区UTR of MTDH. To test whether MTDH expression was regulated by miR-145, we compared MTDH expression in cells with and without miR-145 overexpression. As shown in Figure 5C, both transient and stable ectopic overexpression of miR-145 led to the inhibition of MTDH expression. In contrast, miR-145 inhibitor dose-dependently restored MTDH expression.
To confirm that MTDH is a direct target of miR-145, we then introduced MTDH 3区UTR and corresponding mutant counterparts into pmiRGLO vector. These reporter vectors were then transfected into cells with and without miR-145 overexpression. We found that miR-145 overexpression reduced the luciferase activity in cells transfected with the wild type 3बUTR of MTDH but not in cells with mutant 3区UTR in two cell lines (Figure 5D). Taken together, these results indicate that $\mathrm{MTDH}$ is a direct downstream target of miR-145 and MTDH overexpression in HGSOC may be attributed to the reduced expression of miR-145. 
A
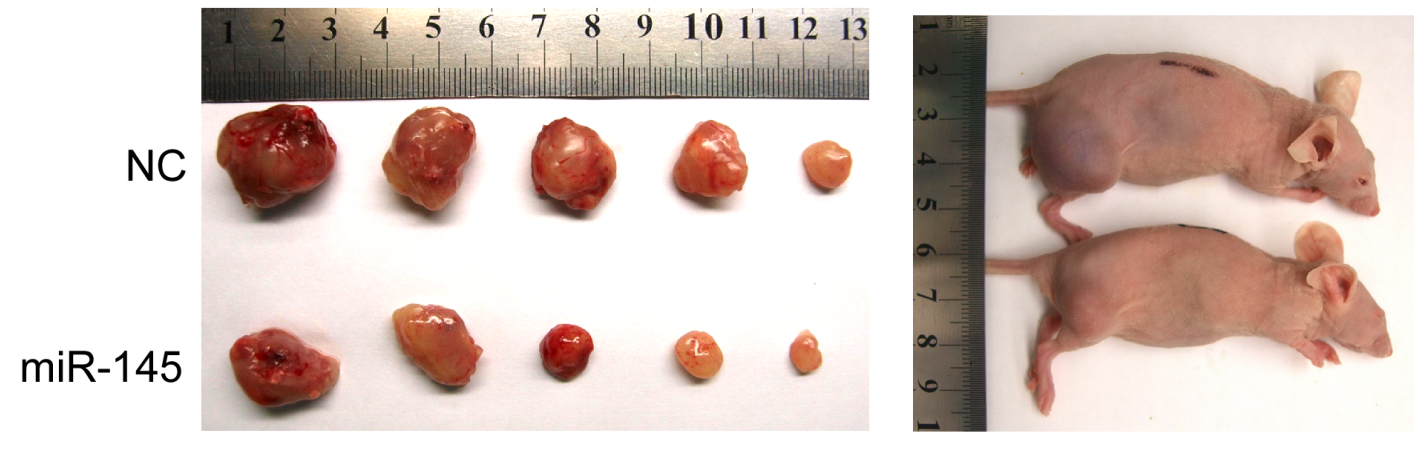

B
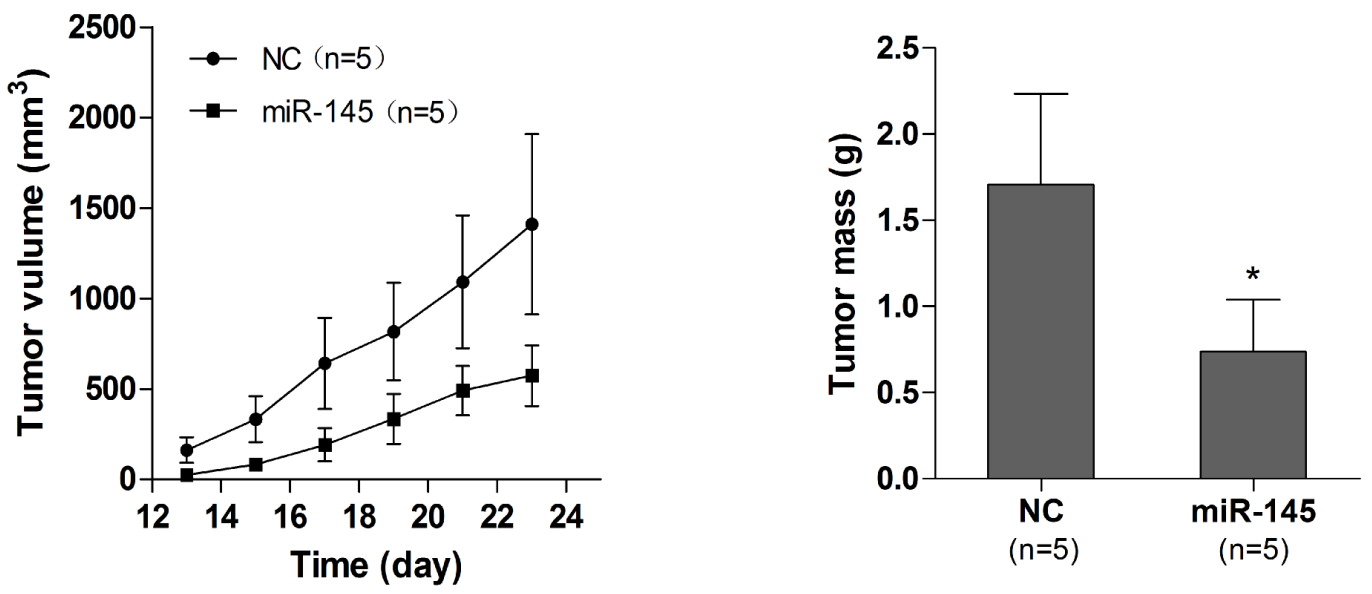

D
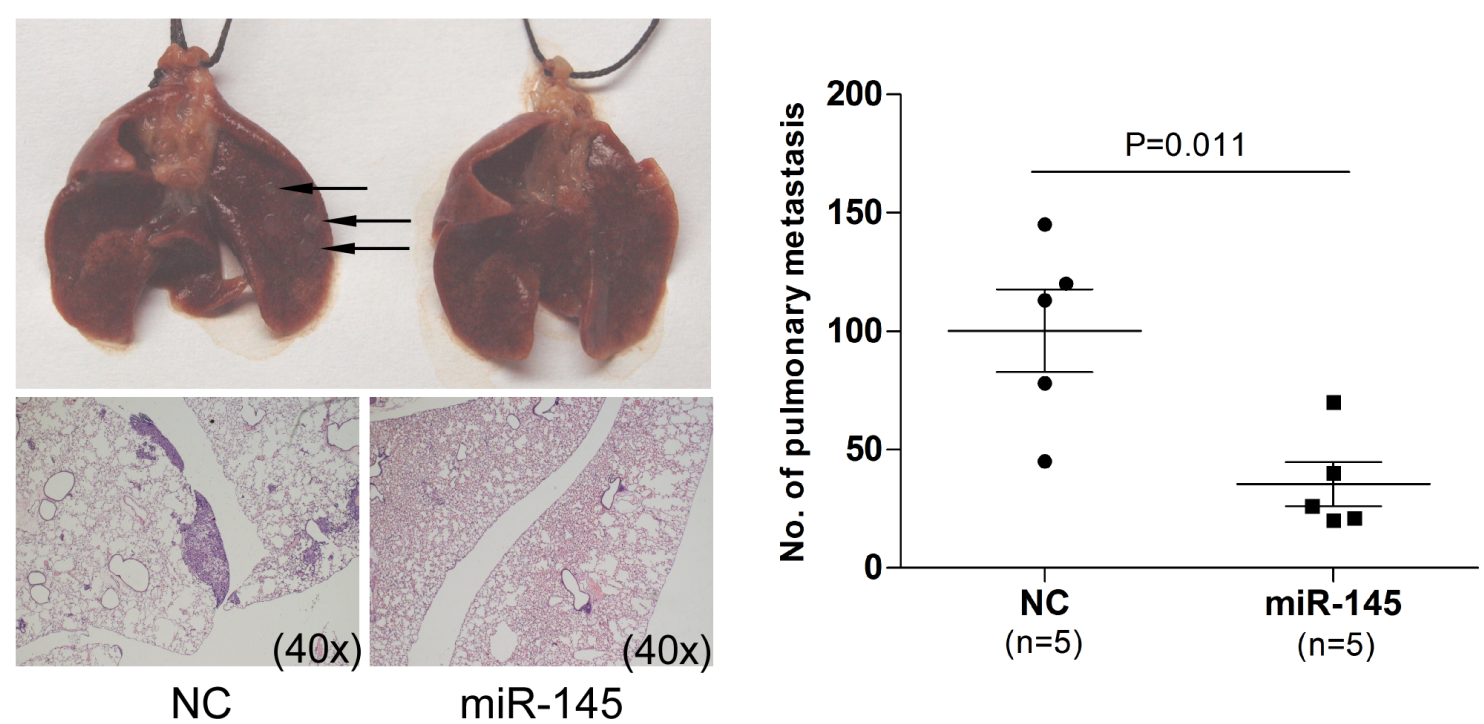

Figure 4: miR-145 overexpression inhibits tumor growth and metastasis in a xenograft model. (A) Photographs illustrate representative tumors in xenografts of A2780 cell lines with miR-145 overexpression (miR-145) compared to tumors without miR-145 overexpression (NC). (B) Growth curves for tumor volumes in xenografts of nude mice with A2780 cell line with and without miR-145 overexpression (each consists of 5 mice), measured from day 13 to day 23. (C) miR-145 overexpression results in a decline of tumor weight. (D) Representative images of lungs (arrows illustrates the visible nodules)and hematoxylin and eosin (HE)-staining of lungs isolated from mice that received tail vein injection of SKOV3 cell line with and without miR-145 overexpression. Each group contains 5 mice. Plot graph illustrates the numbers of pulmonary metastatic nodules under microscope were counted and analyzed with Student's t-test. All data are shown as mean \pm se. $* \mathrm{P}<0.05$. 
A

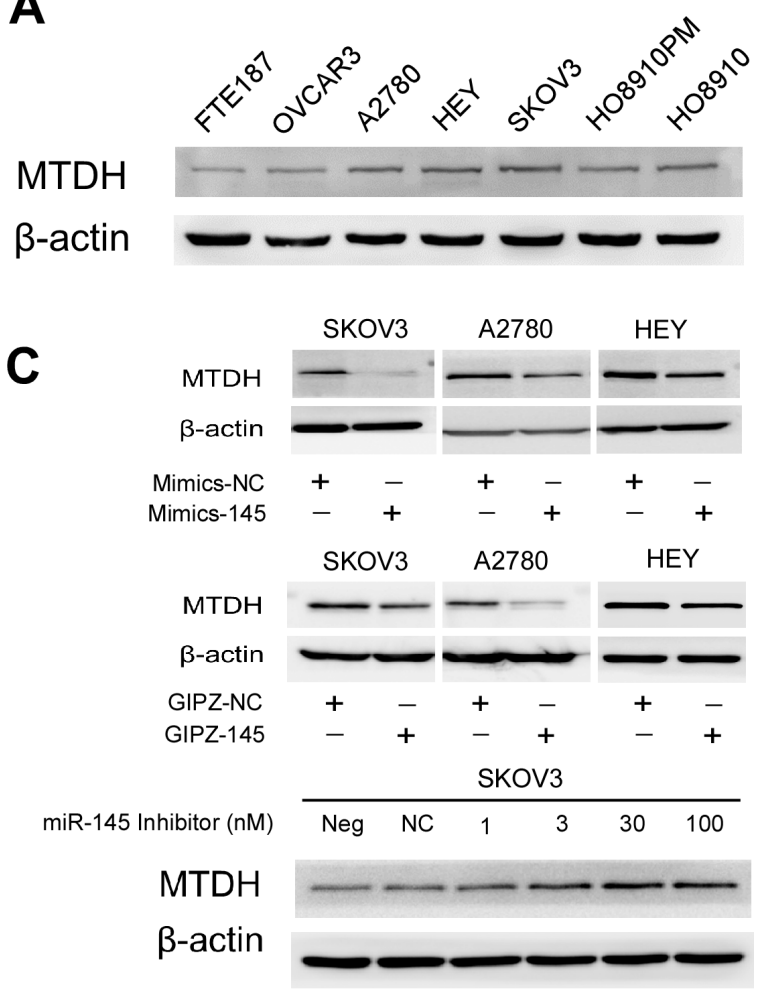

B
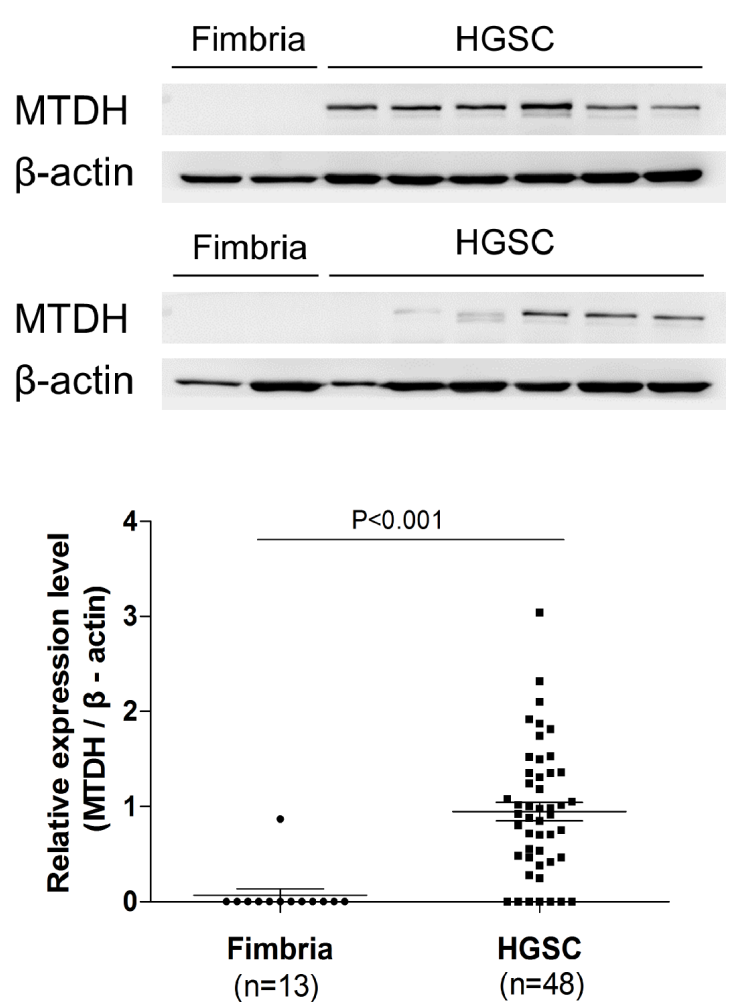

D

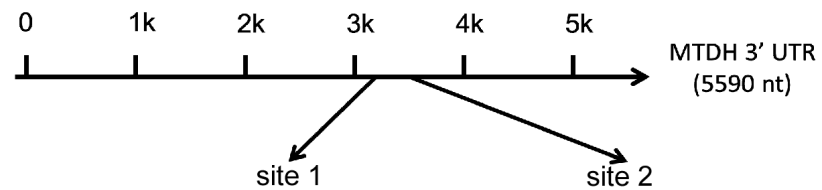

5'...UAGGGGUUUCUCCAGACUGGAAU......UAAACUCACUGCACAAACUGGAA...3' MTDH
WT
11||||
|l| || ||||||
3' UCCCUAAGGACCCUUUUUGACCUG 5' 3' UCCCUAAGGACCCUUUUGACCUG 5' miR-145
MT
5'...UAGGGGUUUCUCCAGCAGAACAU......UAAACUCACUGCACAGCAGAACA...3' MTDH
I |

3' UCCCUAAGGACCCUUUUGACCUG 5' 3' UCCCUAAGGACCCUUUUGACCUG 5' miR-145

SKOV3

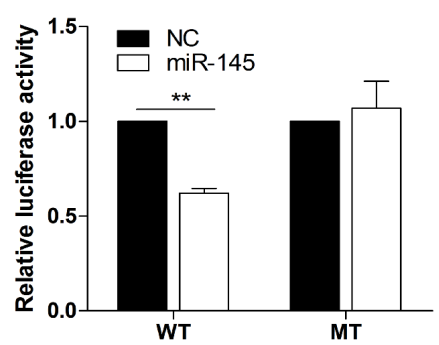

HEY

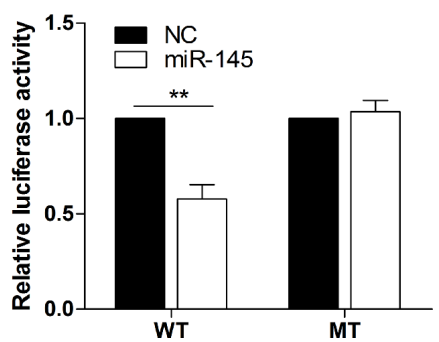

Figure 5: Oncogene MTDH is specifically targeted by miR-145. (A) Western blot analysis of MTDH expression in ovarian cancer cell lines and in normal fallopian tube cell FTE187. (B) Western blot analysis of MTDH in HGSOC tissue samples ( $\mathrm{n}=48$ ) compared to normal fimbria, and the plot graph reveals the statistical result of expression of MTDH in all samples normalized and quantified (mean \pm se). (C) Western blot analysis reveals an inverse correlation of MTDH expression with miR-145 expression in malignant cell lines. Upper panel: transient transfection of miR-145 mimics into cells reduced MTDH protein expression in SKOV3, A2780 and HEY cell lines. Middle panel illustrates that stable overexpression of miR-145 also induced MTDH down-regulation in three cell lines. Lower panel: different concentration of miR-145 inhibitor transfection gradually caused increased expression of MTDH in SKOV3 cell when compared to cell with no transfection (neg) or transfection with inhibitor negative control (NC). (D) Luciferase reporter assay revealed miR-145 suppressed MTDH 3区UTR luciferase activity. The skematic graph shows two predicted binding sites of miR-145 in MTDH 3区UTR and their sequences. Histobars illustrate the relative luciferase activity in SKOV3 and HEY cells with and without miR-145 overexpression when transfected with WT and MT luciferase plasmids (mean \pm se). $* * p<0.01$. 


\section{Overexpression of MTDH rescues the inhibitory effects of miR-145 on ovarian cancer cells}

To explore whether MTDH is involved in miR-145 induced suppression of ovarian cancer cell proliferation and invasion, we performed rescue experiment of MTDH overexpression in miR-145 ectopically expressed cells and corresponding control cells. The MTDH overexpression construct was designed as containing only coding sequences of MTDH without 3区UTR. We used this MTDH overexpressing construct and corresponding vector to transfect A2780 cells with stably overexpressing miR-145 or negative control. We established four stable subgroups of cells and measured the expression level of miR-145 and MTDH by qPCR. As shown in Figure 6A and 6B, we successfully established these four subgroups of cells for rescue experiment. We then attempted to test whether restoration of MTDH could reverse the miR-145 mediated inhibition of metastatic ability of ovarian cancer cells. As can been seen from Figure 6C, overexpression of MTDH with a cDNA without 3凶UTR could partially abrogated miR-145 mediated suppression on migration and invasion of ovarian cancer cells. These observations support that MTDH is one of the major targets mediating the miR-145 effects on tumor metastasis of human ovarian cancer.

\section{p53 represses MTDH through induction of miR-145 in HGSOC}

Based on studies that p53 up-regulates the expression of miR-145[17] and our own finding that MTDH was directly targeted by miR-145, we proposed that p53 may repress MTDH expression through miR-145 in HGSOC. To test this, we measured the expression of p53, miR-145 and MTDH in ovarian cancer cells (with wild type p53 expression) treated with doxorubicin, a p53 activator[18]. As shown in Figure 7A and 7B, miR-145 expression was increased and MTDH expression was decreased when p53 was induced by doxorubicin. These findings suggest that in cells with normal p53, MTDH expression can be indirectly repressed by wild type $\mathrm{p} 53$.

\section{High MTDH expression correlates with poor prognosis of HGSOC patients}

To determine the clinical significance of MTDH in HGSOC, we examined the correlation between MTDH expression in HGSOC tumor samples (Figure 8A) and the clinical outcome $(\mathrm{n}=76)$. The patients were dichotomized into two categories according to their immunoreactivity for MTDH. Then overall survival (OS) and disease-free survival (DFS) rates were estimated by Kaplan-Meier survival curves. As can be seen from Figure 8B, patients with high expression of MTDH had shorter overall survival and disease-free survival period than those with low expression of MTDH. However, only overall survival reached statistical significance $(\mathrm{P}=0.046)$. These findings suggest that MTDH overexpression is a poor prognostic marker in HGSOC patients.

\section{DISCUSSION}

miRNAs were deregulated in ovarian carcinoma[7, 19] and some altered miRNAs were associated with chemoresistance and survival[20, 21]. Here, we demonstrated that miR-145 was frequently down-regulated in HGSOC. We showed that MTDH was directly targeted by miR-145. Furthermore, p53 repressed MTDH through induction of miR-145. Importantly, we found that high MTDH expression level correlated with poor prognosis of HGSOC patients.

miR-145 was down-regulated in many malignancies including ovarian cancer[19]. However, the role of miR-145 in HGSOC remains to be determined. p53 directly and indirectly regulates the expression of a large number of miRNAs including miR-34, miR-200 families as well as miR-145[15]. p53 could also regulate miRNA processing and maturation as for miR-16-1, miR-143 and miR-145[22]. Transcriptional regulation of miR-145 seems to be tissue specific. In mouse, miR-145 and miR-143 are co-transcribed by Srf, myocardin and Nkx2.5 in multipotent cardiac progenitors before becoming localized to smooth muscle cells[23]. In human vascular smooth muscle cell, both TGF- $\beta$ and BMP4 activate transcription of the miR-143/145 gene cluster[24]. In prostate cancer, miR-145 is regulated by DNA methylation and p53 gene mutation [17]. Since TP53 mutations occurred in almost all HGSOC (96\%), down-regulation of miR-145 in HGSOC might be related to $\mathrm{p} 53$ mutation. In this study, p53 activation by doxorubicin caused an up-regulation of miR-145 and a down-regulation of MTDH in ovarian cancer cells (Figure 7A and 7B), which is consistent with the idea that down-regulation of miR-145 may be caused by p53 mutation in HGSOC.

In this study, we identified MTDH as a direct and functional target of miR-145 in HGSOC. Many studies demonstrated that MTDH might play important roles in the pathogenesis, progression, apoptosis regulation, angiogenesis, invasion, metastasis, and overall patient survival in diverse human cancers[25]. A recent study showed that MTDH played a critical role in mammary tumorigenesis by regulating oncogene-induced expansion and activities of tumor-initiating cells (TICs) through interacting with SND1[13]. Multiple mechanisms have been identified to contribute to MTDH overexpression in cancers. Genomic amplification of MTDH has been detected in breast and prostate cancers [16, 26]. Oncogenic Ha-ras could induce MTDH expression through the PI3K-Akt signaling pathway[27]. Other studies showed MTDH was regulated by miRNAs including miR-26a, miR-375, miR-136, and, miR30a[28-30]. Here we identified miR-145 as a new miRNA targeting MTDH.

In summary, we showed that the p53/miR-145 axis regulated MTDH expression in HGSOC. We demonstrate 
A

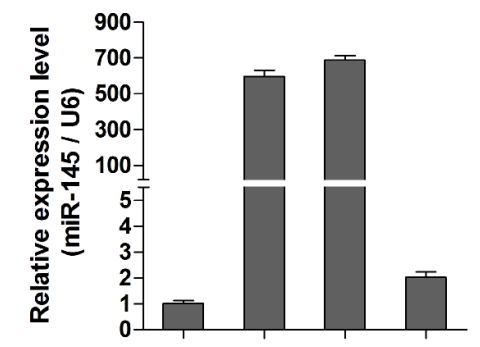

NC

miR-145

Vector

$\mathrm{MTDH}$

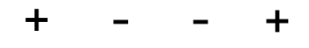

$-++-$

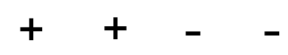

$-\quad+\quad+$
B

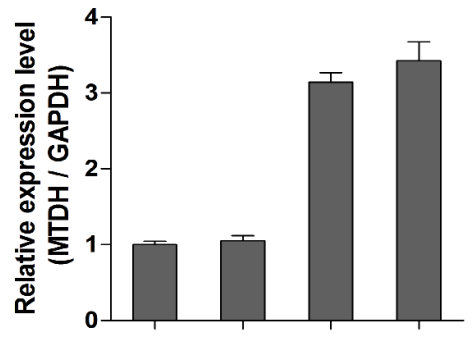

NC

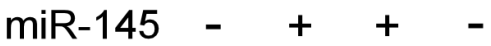

Vector ++-

$\mathrm{MTDH} \quad-\quad-\quad+\quad+$

\section{C}

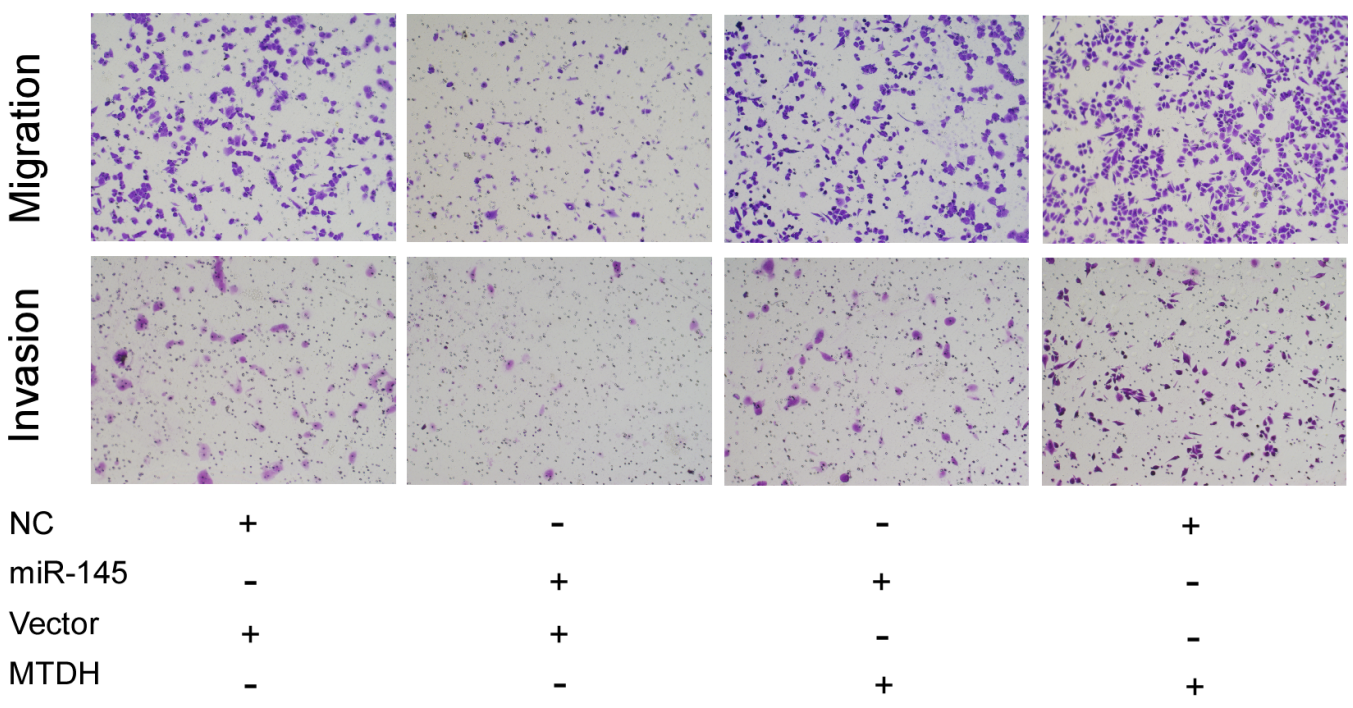

Migration

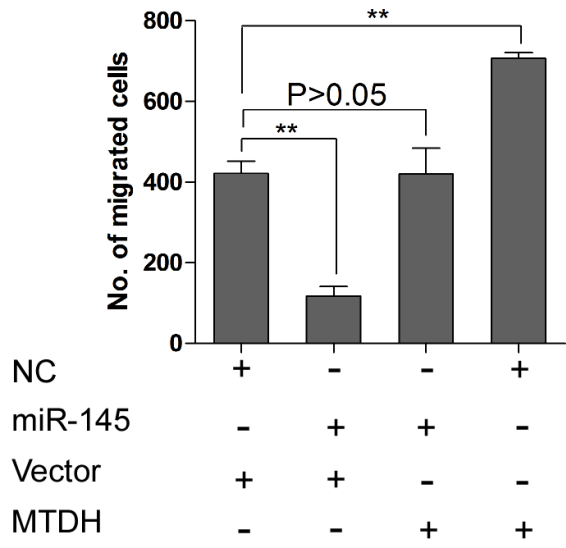

Invasion

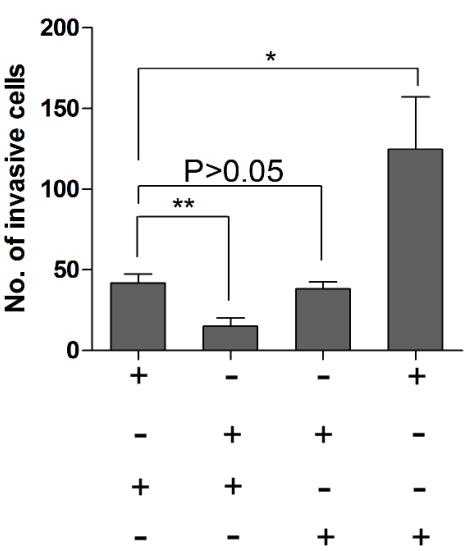

Figure 6: Overexpression of MTDH rescues the inhibitory effects of miR-145 on ovarian cancer cells. (A) QPCR analysis shows expression of miR-145 in cells transfected with different vectors. (B) QPCR analysis shows expression of MTDH in cells transfected with different vectors. MTDH means pBABE-hygro-MTDH overexpression plasmid, and vector is for pBABE-hygro-vector. (C) Transwell assay reveals that reduction of migration and invasion caused by expression of miR-145 can be rescued by introduction of MTDH. ${ }^{*} \mathrm{P}<0.05, * * \mathrm{P}<0.01$. 

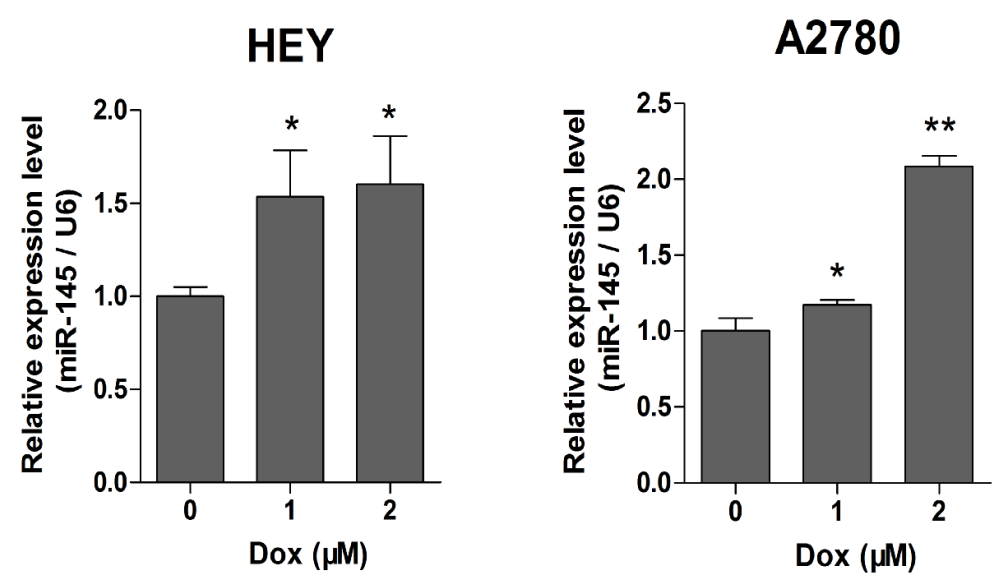

B

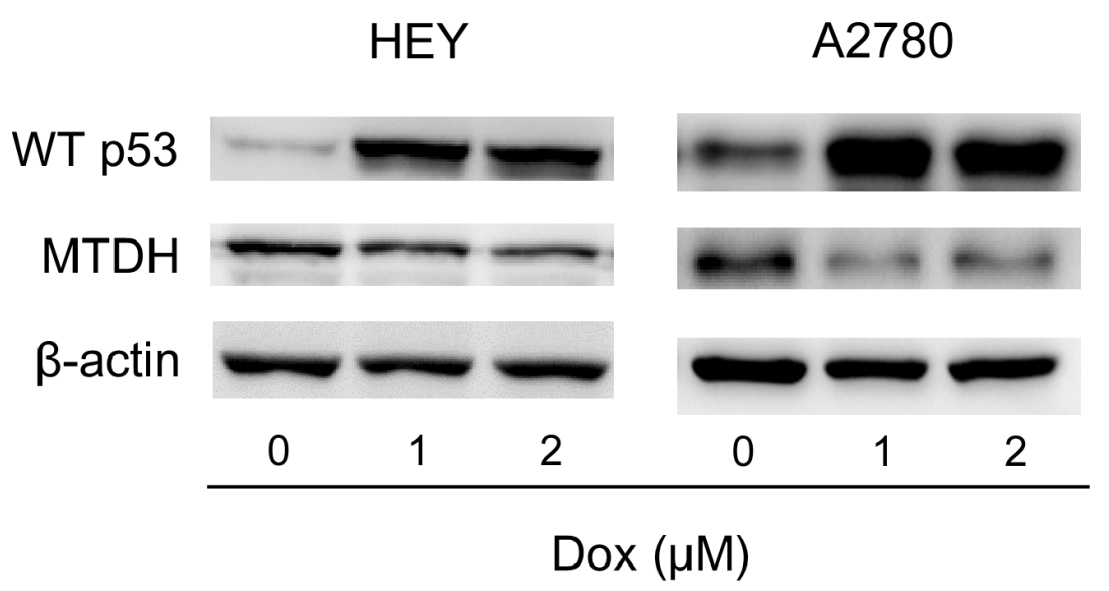

Figure 7: Wild type p53 represses MTDH through induction of miR-145. (A) Doxorubicin (Dox) was applied to induce p53 up-regulation, and qPCR assay shows that miR-145 can be significantly overexpressed after Dox utilization. (B) Western blot analysis of p53 and MTDH expression after treatment of Dox in HEY and A2780 cells. ${ }^{*} \mathrm{P}<0.05,{ }^{*} * \mathrm{P}<0.01$.

that MTDH, which is overexpressed in HGSOC, is directly targeted by miR-145. Importantly, high MTDH expression correlates with poor prognosis of HGSOC patients. Our current study provides a new link between p53, miR-145 and MTDH in the regulation of tumor growth and metastasis in HGSOC. Future studies are needed to assess the therapeutic effect of restoration of miR-145. MTDH can become a powerful prognosis marker for HGSOC.

\section{MATERIALS AND METHODS}

\section{Patients and tissue samples}

High-grade serous ovarian carcinoma (HGSOC) and fallopian tube tissues were collected in Qilu Hospital from April 2008 to July 2012. The HGSOC specimens were obtained from primary ovarian cancer patients receiving no surgery or chemotherapy previously. Fallopian tube tissues were from patients who received a total hysterectomy and bilateral salpingo-oophorectomy for uterine diseases or for benign neoplastic adnexal pathologic changes. All the fresh samples were obtained at surgery, immersed in RNAlater ( Ambion) and stored at $-80^{\circ} \mathrm{C}$. Ethics Committee of Shandong University approved the study and all participants gave written informed consent. The clinicopathologic characteristics were shown in Supplementary Table S2.

\section{Microarray analysis}

Five pairs of collected tissues (fimbria and HGSOC) underwent microarray analysis. Total RNA was harvested using Trizol reagent (Invitrogen) and miRNeasy mini kit (QIAGEN) according to manufacturer's instructions. After having passed RNA quantity measurement using the NanoDrop 1000, the samples were labeled using the miRCURY' ${ }^{\mathrm{TM}} \mathrm{Hy} 3^{\mathrm{TM}} / \mathrm{Hy} 5^{\mathrm{TM}}$ Power labeling kit and hybridized on the miRCURYTM LNA Array (v.18.0). 

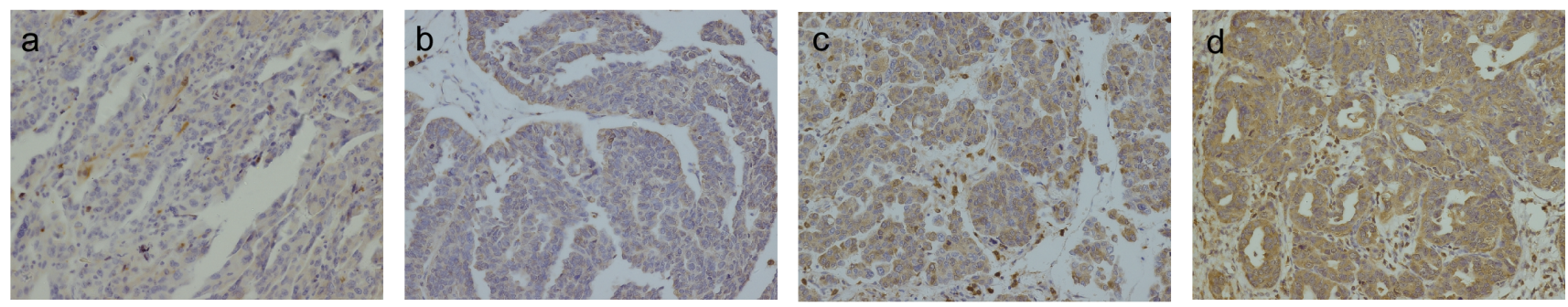

B
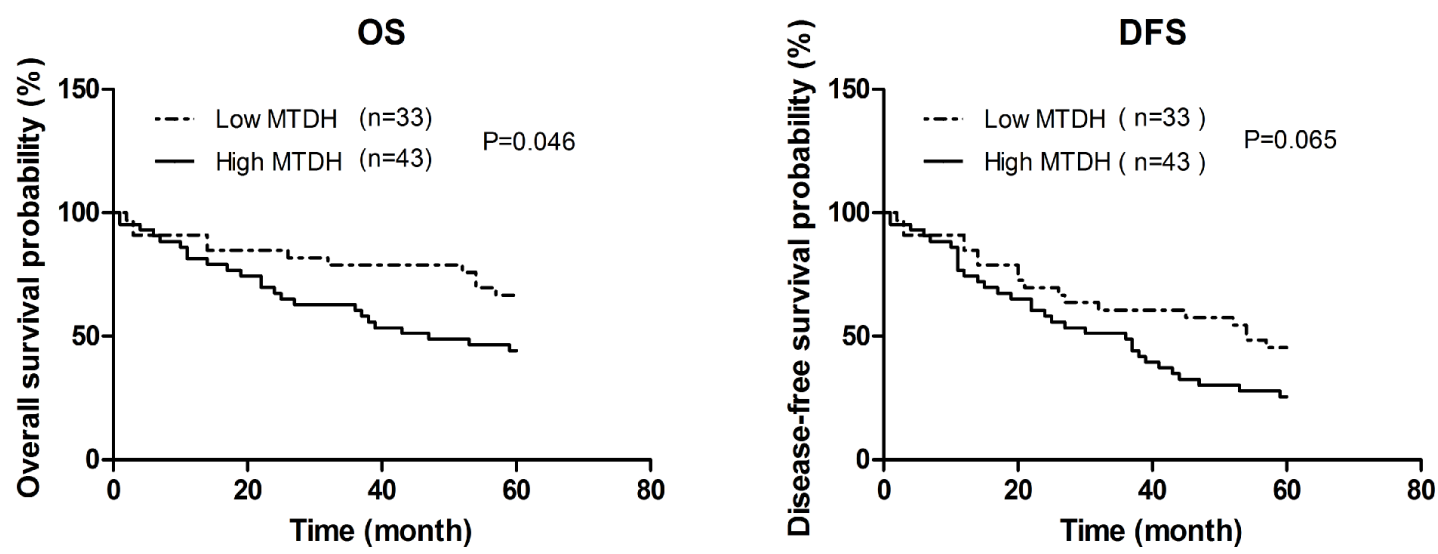

Figure 8: Expression level of MTDH in HGSOC samples correlates with prognosis of patients. (A) Representative images of immunohistochemical analysis of MTDH expression in HGSOC patients samples. (a: $s c o r e=0$, b: $\operatorname{score}=1$, c: $s c o r e=2$, and d: $s c o r e=3$ ). (B) Kaplan-Meier plots of overall survival, and disease-free survival for HGSOC patients dichotomized on the basis of high (score 2 and 3 ) versus low (score 0 and 1) MTDH immunohistochemical expression level.

Following the washing steps the slides were scanned using the Axon GenePix 4000B microarray scanner. Scanned images were then imported into GenePix Pro 6.0 software (Axon) for grid alignment and data extraction. Replicated miRNAs were averaged and miRNAs that intensities $>=30$ in all samples were chosen for calculating normalization factor. Expressed data were normalized using the Median normalization. After normalization, significant differentially expressed miRNAs were identified through Volcano Plot filtering. Finally, hierarchical clustering was performed to show distinguishable miRNA expression profiling among samples. The microarray data generated in this study have been deposited in NCBI GEO database under the accession number GSE61485.

\section{Cell lines and cell culture}

HO8910, HO8910PM, OVCAR3 and HEK293T cells were purchased from China Type Culture Collection (Shanghai,China). FTE187 (immortalized normal human fallopian tube epithelial cell line) cell line was from Jinsong Liu's lab as described[31]. SKOV3, HEY, A2780 and Phoenix amphotropic cells (retroviral packaging cells) were from Jian-Jun Wei's lab as previously described[8].
FTE187 cell line was maintained in cell culture medium consisting of 1:1 Medium199 (Sigma-Aldrich) and MCDB105 medium (Sigma-Aldrich) with 10\% heatinactivated fetal bovine serum (FBS, Gibco) and $10 \mathrm{ng} / \mathrm{ml}$ epidermal growth factor (Sigma-Aldrich). SKOV3, HO8910, OVCAR3 and HO8910PM cells were cultured in RPMI 1640 medium (Gibco). HEK293T, HEY and A2780 cells were cultured in Dulbecco's modified Eagle's medium (DMEM) (Gibco). All media contained $10 \%$ FBS, $100 \mu \mathrm{g} / \mathrm{ml}$ penicillin, and $100 \mu \mathrm{g} / \mathrm{ml}$ streptomycin. All cells were cultured at $37^{\circ} \mathrm{C}$ with $5 \% \mathrm{CO} 2$ in a humidified incubator (Thermo Fisher Scientific).

\section{Plasmid construction and transfection}

To generate a miR-145 expression vector, a $487 \mathrm{bp}$ fragment containing pre-miR-145 was cloned into pGIPZ lentiviral vector (Open Biosystems). Lentivirus expressing miR-145 or negative control (NC) was produced in HEK293T cells packaged by pMD2G and psPAX2. For stable infection, $1 \times 10^{5}$ cells were plated in six-well plates along with $2 \mathrm{~mL}$ of medium without antibiotics. After overnight incubation, the medium was removed and replaced with $1 \mathrm{~mL}$ per well of Opti-MEM 
Reduced-Serum Medium (Gibco) containing $8 \mu \mathrm{g} / \mathrm{mL}$ polybrene. Next, $50 \mu \mathrm{L}$ of concentrated lentiviral particles were added to each well. Twenty-four hours later, fresh medium containing $2 \mu \mathrm{g} / \mathrm{mL}$ puromycin (Sigma-Aldrich) was added to each well. Fresh medium containing puromycin was replaced every 3 to 4 days. Single colonies were obtained after 3 weeks of puromycin selection. For MTDH overexpression, the retroviral expression vector pBabe-hygro was used. pBabe-hygro-MTDH overexpression plasmid was a gift from Dr. Guohong $\mathrm{Hu}$. Viruses were generated by Phoenix amphotropic cells and used to infect target cells as above except that the infected cells were selected with $200 \mathrm{ug} / \mathrm{ml}$ hygromycin (Roche). Primers for plasmid construction are shown in Supplementary Table S3.

\section{Transient transfection}

Mimics (Gene Pharma) and inhibitor (Invitrogen) of miR-145 and the corresponding negative control were used to achieve the transient overexpression or knockdown of miR-145. Cells were transfected with lipofectamine 2000 (Invitrogen) according to the manufacturer's protocol. Transfection was performed at 50nM for mimics and $1-100 \mathrm{nM}$ for inhibitor.

\section{RNA isolation and Quantitative real-time PCR}

Total RNA was extracted from cultured cells or fresh tissues with Trizol reagents (Ambion) according to the manufacturer's protocol. The cDNA of miRNA was synthesized with One Step PrimeScript miRNA cDNA Synthesis Kit (Takara). Quantitative real-time PCR (qPCR) was performed with the SYBR green Premix Ex Taq II (Takara) with StepOne Plus Real-Time PCR System (Applied Biosystems). The expression of U6 was used as the endogenous control for detection of miRNA expression level. Primer information used in the study can also be found in Supplementary Table S3.

\section{Western blot analysis}

Cells were lysed and protein concentration was determined using the BCA Assay Kit (Thermo Scientific). Protein samples were separated by SDS-PAGE and electrotransferred onto PVDF membrane (Millipore). After blocking with 5\% non-fat milk, the membrane was incubated overnight at $4{ }^{\circ} \mathrm{C}$ with the primary antibody and then with horseradish peroxydase-coupled secondary antibody. Signal was detected with enhanced chemiluminescence (ECL) (PerkinElmer) by ImageQuant LAS 4000 (GE Healthcare Life Sciences). Antibodies used include anti-MTDH (Invitrogen), anti-P53 (DAKO), anti- $\beta$-actin (Sigma-Aldrich). Other antibodies used in this study were from Cell signaling Technology.

\section{Migration and invasion assays}

Migration and invasion assays were performed using transwell system (24-well, $8 \mu \mathrm{m}$ pore size; BD falcon) according to manuals. Briefly $5-10 \times 10^{4}$ cells were seeded into the upper chambers of the transwell with certain medium containing no FBS, and lower chambers were filled with culture media containing $20 \% \mathrm{FBS}$ as a chemoattractant. The chambers were incubated at $37^{\circ} \mathrm{C}$ for 4-48 hours depending on the cell lines type. Successfully translocated cells were fixed by methanol for 15 minutes, stained with $0.2 \%$ crystal violet for 30 minutes, and counted under a light microscope.

\section{MTT assay}

The proliferation ability of cells was measured using 3-[4, 5-dimethylthiazol-2-yl]-2, 5-diphenyltetrazolium bromide (MTT) assay. Cells were seeded into 96 -well plates $\left(1.5-3 \times 10^{3}\right.$ cells $/$ well $)$ for continual $1-5$ days. At specified time points, $20 \mu \mathrm{l}$ of MTT (Sigma-Aldrich) solution $(0.5 \mathrm{mg} / \mathrm{ml})$ was added to each well, and the cells were incubated for additional 4 hours at $37^{\circ} \mathrm{C}$. Then the supernatants were carefully removed and $100 \mu$ l of dimethyl sulfoxide (DMSO, Sigma-Aldrich) was added to each well. The absorbance values were evaluated at $490 \mathrm{~nm}$ with a Microplate Reader (Thermo Fisher Scientific).

\section{Clonogenic assay}

Clonogenic assay was performed as described previously[32]. Briefly, single-cell suspensions were generated for each cell line and specified numbers of cells were seeded into six-well tissue culture plates. Then cells were cultured for 10 days. Colonies were stained with crystal violet. Colonies of greater than 50 cells were counted. The data presented is the mean \pm standard error (SE) and represents three independent experiments.

\section{Luciferase reporter assay}

The 3区UTR of MTDH containing two putative miR-145 binding sites (580bp) was amplified and cloned into pmirGLO vector (Promega) using the SacI and XbaI sites to generate the wild type construct [1]. For mutant plasmid (MT), overlap extension PCR assay [33] was used as described previously [34]. Cells were cultured in 96-well plates and transfected with 100ng of WT or MT MTDH 3बUTR constructs by lipofectamine 2000 assay. Twenty-four hours after transfection, luciferase activity was measured using the Dual-Glo Luciferase Assay System (Promega). Renilla luciferase activity was normalized to corresponding firefly luciferase activity and plotted as a percentage. 


\section{In vivo nude mice tumorigenesis and metastasis assay}

For xenograft experiment, $5 \times 10^{6}$ cells in $120 \mu \mathrm{l}$ mixed solution (PBS: Matrigel $(\mathrm{BD}$ falcon $)=5: 1$ ) were injected subcutaneously into 4-5 weeks-old BALB/c $\mathrm{nu} / \mathrm{nu}$ female mice. Tumor growth was monitored by measuring tumor diameters. Tumor volume was calculated according to the formula $\mathrm{TV}(\mathrm{cm} 3)=\mathrm{a} \times \mathrm{b} 2 \times \pi / 6$, where $a$ is the longest diameter, and $b$ is the shortest diameter. Mouse was euthanized when a tumor reached $2 \mathrm{~cm}$ in diameter. To produce experimental lung metastasis, $2 \times 10^{6}$ cells in $100 \mu \mathrm{l}$ PBS were injected into the lateral tail veins of 4-5 weeks-old $\mathrm{BALB} / \mathrm{c} \mathrm{nu} / \mathrm{nu}$ female mice. 6 weeks later, mice were sacrificed under anesthesia. The lungs were collected and fixed in 10\% formalin. For tissue morphology evaluation, hematoxylin and eosin (HE) staining was performed on sections from embedded samples. All animal experiments were performed with the approval of Shandong University Animal Care and Use Committee.

\section{Immunohistochemistry}

Formalin-fixed and paraffin-embedded tissues were sectioned at $4 \mu \mathrm{m}$. Tissue slides were deparaffinized in xylene and rehydrated in a graded series of ethanol. Antigen retrieval was performed by heat-induced epitope retrieval. All immunohistochemical staining procedures were performed on a Ventana Nexus automated system. After blocking in 1.5\% normal goat serum for 30 minutes at room temperature, slides were then incubated overnight at $4^{\circ} \mathrm{C}$ with primary antibodies of MTDH (Invitrogen ) in a humid chamber. Staining was detected with I-View 3,3'-diaminobenzidine (DAB) detection system. MTDH staining results were scored based on staining intensity $(0=$ negative, $1=$ weak, $2=$ moderate, $3=$ strong).

\section{Statistics analysis}

The software SPSS V20.0 was used for statistical analysis. Student's t-test and one-way ANOVA analysis were used to determine significance. $\mathrm{P}<0.05$ was considered statistically significant.

\section{Conflicts of interest}

No potential conflicts of interest were disclosed.

\section{ACKNOWLEDGEMENTS}

This study was partially supported by awards from National 863 Program (2014AA020605), National Basic Research Program of China (973 Program, 2011CB966200), and The National Natural Science Foundation of China $(81272857,81171897)$.

\section{REFERENCES}

1. Vaughan S, Coward JI, Bast RC, Jr., Berchuck A, Berek JS, Brenton JD, Coukos G, Crum CC, Drapkin R, Etemadmoghadam D, Friedlander M, Gabra H, Kaye SB, Lord CJ, Lengyel E, Levine DA, et al. Rethinking ovarian cancer: recommendations for improving outcomes. Nature reviews Cancer. 2011; 11:719-725.

2. Vang R, Shih Ie M, Kurman RJ. Ovarian low-grade and highgrade serous carcinoma: pathogenesis, clinicopathologic and molecular biologic features, and diagnostic problems. Adv Anat Pathol. 2009; 16:267-282.

3. Cancer Genome Atlas Research N . Integrated genomic analyses of ovarian carcinoma. Nature. 2011; 474:609-615.

4. Perets R, Wyant GA, Muto KW, Bijron JG, Poole BB, Chin KT, Chen JY, Ohman AW, Stepule CD, Kwak S, Karst AM, Hirsch MS, Setlur SR, Crum CP, Dinulescu DM, Drapkin R. Transformation of the fallopian tube secretory epithelium leads to high-grade serous ovarian cancer in Brca;Tp53;Pten models. Cancer cell. 2013; 24:751-765.

5. Di Leva G, Croce CM. The Role of microRNAs in the Tumorigenesis of Ovarian Cancer. Front Oncol. 2013; 3:153.

6. Korpal M, Ell BJ, Buffa FM, Ibrahim T, Blanco MA, Celia-Terrassa T, Mercatali L, Khan Z, Goodarzi H, Hua Y, Wei Y, Hu G, Garcia BA, Ragoussis J, Amadori D, Harris AL, et al. Direct targeting of Sec23a by miR-200s influences cancer cell secretome and promotes metastatic colonization. Nat Med. 2011; 17:1101-1108.

7. Iorio MV, Visone R, Di Leva G, Donati V, Petrocca F, Casalini P, Taccioli C, Volinia S, Liu CG, Alder H, Calin GA, Menard S, Croce CM. MicroRNA signatures in human ovarian cancer. Cancer Res. 2007; 67:8699-8707.

8. Liu Z, Liu J, Segura MF, Shao C, Lee P, Gong Y, Hernando E, Wei JJ. MiR-182 overexpression in tumourigenesis of high-grade serous ovarian carcinoma. J Pathol. 2012; 228:204-215.

9. Gotte M, Mohr C, Koo CY, Stock C, Vaske AK, Viola M, Ibrahim SA, Peddibhotla S, Teng YH, Low JY, Ebnet K, Kiesel L, Yip GW. miR-145-dependent targeting of junctional adhesion molecule $\mathrm{A}$ and modulation of fascin expression are associated with reduced breast cancer cell motility and invasiveness. Oncogene. 2010; 29: 6569-6580.

10. Sachdeva M, Zhu S, Wu F, Wu H, Walia V, Kumar S, Elble R, Watabe K, Mo YY. p53 represses c-Myc through induction of the tumor suppressor miR-145. Proc Natl Acad Sci U S A. 2009; 106:3207-3212.

11. Gao P, Xing AY, Zhou GY, Zhang TG, Zhang JP, Gao C, Li H, Shi DB. The molecular mechanism of microRNA-145 to suppress invasion-metastasis cascade in gastric cancer. Oncogene. 2013; 32:491-501.

12. $\mathrm{Xu}$ Q, Liu LZ, Qian X, Chen Q, Jiang Y, Li D, Lai L, Jiang BH. MiR-145 directly targets p70S6K1 in cancer cells to inhibit tumor growth and angiogenesis. Nucleic Acids Res. 2012; 40:761-774. 
13. Wan L, Lu X, Yuan S, Wei Y, Guo F, Shen M, Yuan M, Chakrabarti R, Hua Y, Smith HA, Blanco MA, Chekmareva M, Wu H, Bronson RT, Haffty BG, Xing Y, et al. MTDH-SND1 Interaction Is Crucial for Expansion and Activity of Tumor-Initiating Cells in Diverse Oncogene- and Carcinogen-Induced Mammary Tumors. Cancer Cell. 2014; 26:92-105.

14. Li C, Li Y, Wang X, Wang Z, Cai J, Wang L, Zhao Y, Song H, Meng X, Ning X, Xu C, Lin M, Li L, Geng J. Elevated expression of astrocyte elevated gene-1 (AEG-1) is correlated with cisplatin-based chemoresistance and shortened outcome in patients with stages III-IV serous ovarian carcinoma. Histopathology. 2012; 60:953-963.

15. Hermeking H. MicroRNAs in the p53 network: micromanagement of tumour suppression. Nature reviews Cancer. 2012; 12:613-626.

16. Hu G, Chong RA, Yang Q, Wei Y, Blanco MA, Li F, Reiss M, Au JL, Haffty BG, Kang Y. MTDH activation by 8 q22 genomic gain promotes chemoresistance and metastasis of poor-prognosis breast cancer. Cancer Cell. 2009; 15:9-20.

17. Suh SO, Chen Y, Zaman MS, Hirata H, Yamamura S, Shahryari V, Liu J, Tabatabai ZL, Kakar S, Deng G, Tanaka Y, Dahiya R. MicroRNA-145 is regulated by DNA methylation and p53 gene mutation in prostate cancer. Carcinogenesis. 2011; 32:772-778.

18. Xu Y, Diao Y, Qi S, Pan X, Wang Q, Xin Y, Cao X, Ruan J, Zhao Z, Luo L, Liu C, Yin Z. Phosphorylated Hsp27 activates ATM-dependent p53 signaling and mediates the resistance of MCF-7 cells to doxorubicin-induced apoptosis. Cell Signal. 2013; 25:1176-1185.

19. Nam EJ, Yoon H, Kim SW, Kim H, Kim YT, Kim JH, Kim JW, Kim S. MicroRNA expression profiles in serous ovarian carcinoma. Clin Cancer Res. 2008; 14:2690-2695.

20. Hu X, Macdonald DM, Huettner PC, Feng Z, El Naqa IM, Schwarz JK, Mutch DG, Grigsby PW, Powell SN, Wang X. A miR-200 microRNA cluster as prognostic marker in advanced ovarian cancer. Gynecol Oncol. 2009; 114: 457-464.

21. van Jaarsveld MT, Helleman J, Boersma AW, van Kuijk PF, van Ijcken WF, Despierre E, Vergote I, Mathijssen RH, Berns EM, Verweij J, Pothof J, Wiemer EA. miR-141 regulates KEAP1 and modulates cisplatin sensitivity in ovarian cancer cells. Oncogene. 2013; 32:4284-4293.

22. Suzuki HI, Yamagata K, Sugimoto K, Iwamoto T, Kato S, Miyazono K. Modulation of microRNA processing by p53. Nature. 2009; 460:529-533.

23. Cordes KR, Sheehy NT, White MP, Berry EC, Morton SU, Muth AN, Lee TH, Miano JM, Ivey KN, Srivastava D. miR-145 and miR-143 regulate smooth muscle cell fate and plasticity. Nature. 2009; 460:705-710.
24. Davis-Dusenbery BN, Chan MC, Reno KE, Weisman AS, Layne MD, Lagna G, Hata A. down-regulation of Kruppellike factor-4 (KLF4) by microRNA-143/145 is critical for modulation of vascular smooth muscle cell phenotype by transforming growth factor-beta and bone morphogenetic protein 4. J Biol Chem. 2011; 286:28097-28110.

25. Emdad L, Das SK, Dasgupta S, Hu B, Sarkar D, Fisher PB. AEG-1/MTDH/LYRIC: signaling pathways, downstream genes, interacting proteins, and regulation of tumor angiogenesis. Adv Cancer Res. 2013; 120:75-111.

26. Wan L, Hu G, Wei Y, Yuan M, Bronson RT, Yang Q, Siddiqui J, Pienta KJ, Kang Y. Genetic Ablation of Metadherin Inhibits Autochthonous Prostate Cancer Progression and Metastasis. Cancer Res. 2014; .

27. Lee SG, Su ZZ, Emdad L, Sarkar D, Fisher PB. Astrocyte elevated gene-1 (AEG-1) is a target gene of oncogenic Ha-ras requiring phosphatidylinositol 3-kinase and c-Myc. Proc Natl Acad Sci U S A. 2006; 103:17390-17395.

28. Nohata N, Hanazawa T, Kikkawa N, Mutallip M, Sakurai D, Fujimura L, Kawakami K, Chiyomaru T, Yoshino H, Enokida H, Nakagawa M, Okamoto Y, Seki N. Tumor suppressive microRNA-375 regulates oncogene AEG-1/MTDH in head and neck squamous cell carcinoma (HNSCC). J Hum Genet. 2011; 56:595-601.

29. Zhang B, Liu XX, He JR, Zhou CX, Guo M, He M, Li MF, Chen GQ, Zhao Q. Pathologically decreased miR-26a antagonizes apoptosis and facilitates carcinogenesis by targeting MTDH and EZH2 in breast cancer. Carcinogenesis. 2011; 32:2-9.

30. Yang Y, Wu J, Guan H, Cai J, Fang L, Li J, Li M. MiR-136 promotes apoptosis of glioma cells by targeting AEG-1 and Bcl-2. FEBS Lett. 2012; 586:3608-3612.

31. Shan W, Mercado-Uribe I, Zhang J, Rosen D, Zhang S, Wei J, Liu J. Mucinous adenocarcinoma developed from human fallopian tube epithelial cells through defined genetic modifications. Cell cycle (Georgetown, Tex. 2012; 11:2107-2113.

32. Mueller S, Yang X, Sottero TL, Gragg A, Prasad G, Polley MY, Weiss WA, Matthay KK, Davidoff AM, DuBois SG, Haas-Kogan DA. Cooperation of the HDAC inhibitor vorinostat and radiation in metastatic neuroblastoma: efficacy and underlying mechanisms. Cancer letters. 2011; 306:223-229.

33. Ho SN, Hunt HD, Horton RM, Pullen JK, Pease LR. Site-directed mutagenesis by overlap extension using the polymerase chain reaction. Gene. 1989; 77:51-59.

34. Wei JJ, Wu X, Peng Y, Shi G, Basturk O, Yang X, Daniels G, Osman I, Ouyang J, Hernando E, Pellicer A, Rhim JS, Melamed J, Lee P. Regulation of HMGA1 expression by microRNA-296 affects prostate cancer growth and invasion. Clin Cancer Res. 2011; 17:1297-1305. 